\title{
Physician-based availability of psychotherapy in Ontario: a population-based retrospective cohort study
}

\author{
Paul Kurdyak MD PhD, Juveria Zaheer MD MSc, André Carvalho MD PhD, Claire de Oliveira PhD, \\ Michael Lebenbaum MSc, Andrew S. Wilton MSc, Mark Fefergrad MD, Vicky Stergiopoulos MD MSc, \\ Benoit H. Mulsant MD MS
}

Abstract

Background: Psychotherapy is recommended as a first-line treatment for the management of common psychiatric disorders. The objective of this study was to evaluate the availability of publicly funded psychotherapy provided by physicians in Ontario by describing primary care physicians (PCPs) and psychiatrists whose practices focus on psychotherapy and comparing them to PCPs and psychiatrists whose practices do not.

Methods: This was a population-based retrospective cohort study. We included all PCPs and psychiatrists in Ontario who submitted at least 1 billing claim to the Ontario Health Insurance Plan between Apr. 1, 2015, and Mar. 31, 2016, and categorized them as psychotherapists if at least $50 \%$ of their outpatient billings were related to the provision of psychotherapy. We measured practice characteristics such as total number of patients and new patients, and average visit frequency for 4 physician categories: PCP nonpsychotherapists, PCP psychotherapists, psychiatrist nonpsychotherapists and psychiatrist psychotherapists. We also measured access to care for people with urgent need for mental health services.

Results: Of 12772 PCPs, 404 (3.2\%) were PCP psychotherapists; of 2150 psychiatrists, 586 (27.3\%) were psychotherapists. Primary care physician nonpsychotherapists had the highest number of patients and number of new patients, followed by psychiatrist nonpsychotherapists, PCP psychotherapists and psychiatrist psychotherapists. Primary care physician nonpsychotherapists had the lowest average annual number of visits per patient, whereas both types of psychotherapists had a much greater number of visits per patient. Primary care physician and psychiatrist nonpsychotherapists saw about $25 \%$ of patients with urgent needs for mental health services, whereas PCP and psychiatrist psychotherapists saw $1 \%-3 \%$ of these patients.

Interpretation: Physicians who provide publicly funded psychotherapy in Ontario see a small number of patients, and they see few of those with urgent need for mental health services. Our findings suggest that improving access to psychotherapy will require the development of alternative strategies.

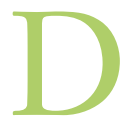

epressive and anxiety disorders are the most common psychiatric disorders; from a public health perspective, they are associated with the highest burden of disability. ${ }^{1,2}$ Evidence-based treatment guidelines for these common psychiatric disorders recommend brief structured psychotherapies as first-line treatment options, particularly for mild to moderate disorders. ${ }^{3-5}$ In Ontario, access to publicly funded psychotherapy is provided mostly by primary care physicians (PCPs) with psychotherapy-focused practices and psychiatrists. A PCP psychotherapist (sometimes referred to as a general practitioner psychotherapist) is a primary care physician who has a practice focused on psychotherapy. There is an opportunity for training, ${ }^{6}$ but no certification or specific training is required to become a PCP psychotherapist.
In August 2018, the Coalition of Ontario Psychiatrists asserted there was a "crisis in access to mental health services" in Ontario, and they attributed this crisis to a shortage of psychiatrists. ${ }^{7}$ They made 3 recommendations, all with the goal of increasing the number of psychiatrists: to increase exposure to psychiatry in medical school, to train more psychiatrists and to increase the payment for psychiatrists' services. However,

Competing interests: None declared.

This article has been peer reviewed.

Correspondence to: Paul Kurdyak, paul.kurdyak@camh.ca

CMAJ Open 2020. DOI:10.9778/cmajo.20190094 
the existing supply and practice patterns of physician psychotherapists is currently unknown. It is critical to know the existing human resource capacity of physician psychotherapists in Ontario in order to better understand the availability of resources to meet demand for psychotherapy.

To address this question, we conducted a study of the existing physician-based availability of psychotherapy in Ontario. We categorized all Ontario PCPs and psychiatrists as psychotherapists or nonpsychotherapists, which resulted in 4 physician categories. We characterized the practices of physicians within these 4 categories and determined whether patients with urgent need for mental health services were seen within 30 or 180 days of the index event by physicians from each of these 4 groups.

\section{Methods}

We conducted a population-based retrospective cohort study of physicians in Ontario between Apr. 1, 2015, and Mar. 31, 2016. We categorized physicians as psychotherapists if at least $50 \%$ of their outpatient billings were related to the provision of psychotherapy. The Ontario Health Insurance Plan billing codes used to capture psychotherapy are listed in Appendix 1, Supplemental Table S1 (available at www.cmajopen.ca/ content/8/1/E105/suppl/DC1). We then compared practice characteristics between 4 physician categories: PCP nonpsychotherapists, PCP psychotherapists, psychiatrist nonpsychotherapists and psychiatrist psychotherapists.

\section{Data sources}

We obtained PCP and psychiatrist characteristics from the ICES Physician Database. We obtained physician practice patterns and the characteristics of their patients from the Ontario Health Insurance Plan database (outpatient billing information), National Ambulatory Care Reporting System (information on emergency department visits), Ontario Mental Health Reporting System and Canadian Institute for Health Information Discharge Abstract Database (information on psychiatric hospital admissions), Registered Persons Database (sociodemographic characteristics) and Immigration, Refugees and Citizenship Canada (immigration data).

These data sets were linked by means of unique encoded identifiers and analyzed at ICES. They are routinely used for research purposes. The universal access to physician and hospital care in Canada means that information is captured routinely when people use health care services (e.g., via physician billings, and information captured systematically for emergency department visits and hospital stays).

The Immigration, Refugees and Citizenship Canada database includes application records for people who applied to land in Ontario. The current data set contains data from 1985 to May 31, 2017. The linkage rate (proportion of applications that were linked successfully to the health administrative data held at ICES) between 1985 and 2012 was $86.4 \% .^{8}$

\section{Study sample}

Primary care physicians and psychiatrists who submitted at least 1 billing claim to the Ontario Health Insurance Plan between Apr. 1, 2015, and Mar. 31, 2016, were included. We focused on outpatients who were provided care and excluded all billings related to inpatient care for both PCPs and psychiatrists.

\section{Physician characteristics}

We assessed the following physician characteristics: sex, age, medical school attended and region of practice based on Local Health Integration Network (LHIN). Local Health Integration Networks were categorized as high supply (Toronto Central and Champlain), medium supply (South West, South East and Hamilton Niagara Haldimand Brant) or low supply (the 9 remaining LHINs). ${ }^{9}$ These supply categories are based on whether the per capita supply of psychiatrists is much higher than the provincial average (high supply), at or near the provincial average (medium supply), or below the provincial average (low supply) ${ }^{9}$ (Appendix 1, Supplemental Figure S1). We also determined whether physicians were full-time using the Canadian Institute for Health Information definition: having annual billings above the 30th percentile for all Ontario PCPs or psychiatrists, respectively. ${ }^{10}$ Measuring the proportion of full-time physicians in each category accounts for variability in the capacity for physicians to provide patient care.

For each physician, we measured the number of unique outpatients, new outpatients (defined as no visits in the $12 \mathrm{mo}$ before the first visit in the study period) and mean number of annual visits per patient. Finally, we measured the number of physicians in each of the 4 physician categories with fewer than 200, 100 and 40 patients seen annually.

\section{Patient characteristics}

We measured characteristics of outpatients seen by physicians in the 4 categories. If a patient was seen by more than 1 physician of a given category during the study period, he or she was assigned to the physician with the highest visit frequency for that patient within each of the 4 categories. We measured the following patient characteristics: sex, age, neighbourhood income quintile based on 2011 census information and residential region (using the same categories as for physicians).

We also assessed the proportions of patients who experienced any of the following events in the 24 months before Apr. 1, 2015: a mental health and addictions admission, an emergency department visit for a mental health reason, an emergency department visit for self-harm, or 2 or more emergency department visits for substance use.

\section{Access to physicians for patients with urgent need for mental health services}

We measured the number of patients who had a mental health and addictions admission, emergency department visit for a mental health reason, emergency department visit for self-harm, or 2 or more emergency department visits for substance use between Apr. 1, 2014, and Mar. 31, 2016. These patient groups are representative of patients in urgent need of mental health services. For patients seen twice in the emergency department for substance use, we used the second visit 
to measure access to care. For the 3 other patient groups, we used the emergency department or hospital discharge date. These 4 patient groups were not mutually exclusive.

We assessed whether patients from these groups were seen by physicians in each of the 4 categories within 30 and 180 days. If a patient saw more than 1 type of physician, each of the physicians seen was credited for the visit. Visits to PCP nonpsychotherapists were determined to be related to mental health and addictions based on a previously validated algorithm. ${ }^{11}$

\section{Statistical analysis}

For each variable, we examined the mean and standard deviation, median and interquartile ratio, or number and proportion. We used standardized differences to assess differences between psychotherapists and nonpsychotherapists separately for PCPs and for psychiatrists. With large samples, $p$ values are often significant with very small differences, whereas a standardized difference is less sensitive to sample size. A standardized difference of greater than 0.1 is considered clinically significant. ${ }^{12}$ We used SAS Version 9 (SAS Institute) for all statistical analyses.

\section{Ethics approval}

The use of data in this study was authorized under section 45 of Ontario's Personal Health Information Protection Act, which does not require review by a research ethics board.

\section{Results}

\section{Physician characteristics}

Between Apr. 1, 2015, and Mar. 31, 2016, there were 12772 primary care physicians who were clinically active in Ontario, of whom 404 (3.2\%) met our criteria as PCP psychotherapists and $12368(96.8 \%)$ as PCP nonpsychotherapists. During the same time period, there were 2150 psychiatrists in Ontario, of whom $586(27.3 \%)$ were psychiatrists who met our criteria for psychotherapist and 1564 (72.7\%) who were psychiatrist nonpsychotherapists. The demographic and regional characteristics, practice patterns and medical school of the 4 physician categories are presented in Table 1 .

Both PCP and psychiatrist psychotherapists were about 9-10 years older than their nonpsychotherapist colleagues (Table 1), and a higher proportion of both types of psychotherapists were in the oldest age category ( $\geq 60 \mathrm{yr}$ ). A smaller proportion of PCP psychotherapists (54.0\%) met the definition of a full-time physician than the other 3 categories of physicians, which were all about $70 \%$ full-time. More than a quarter of both types of psychotherapists graduated from the University of Toronto medical school, whereas a higher proportion of nonpsychotherapist physicians graduated from non-Canadian medical schools. Both categories of psychiatrists were more likely than PCP nonpsychotherapists to practise in high-supply LHINs. Primary care physician and psychiatrist psychotherapists were more likely than their nonpsychotherapist counterparts to practise in high-supply LHINs.

\section{Physician practice characteristics}

The total numbers of outpatients and new outpatients were highest for PCP nonpsychotherapists, followed by psychiatrist nonpsychotherapists, PCP psychotherapists and psychiatrist psychotherapists (Table 2). The visit frequency followed the reverse pattern, with psychiatrist psychotherapists having the highest visit frequencies, and PCP nonpsychotherapists having the lowest. This pattern of patient number and visit frequency translated into 143 (24.4\%), 79 (19.6\%), 201 (12.8\%) and $525(4.2 \%)$ psychiatrist psychotherapists, PCP psychotherapists, psychiatrist nonpsychotherapists and PCP nonpsychotherapists, respectively, who saw fewer than 40 patients annually. The average annual visit frequency for nonpsychotherapist physicians was 3 or fewer, whereas the average visit frequency for psychotherapist physicians was much higher, as high as 40 in some cases. A total of $9.8 \%$ of visits to PCP nonpsychotherapists were related to mental health and addictions admissions.

\section{Patient characteristics}

The patients of both types of psychotherapists were slightly more likely to be female than those of nonpsychotherapists (Table 3). The mean age of patients across the 4 physician categories was similar. Psychotherapists saw a slightly higher proportion of patients from high-income quintile neighbourhoods, and psychiatrist nonpsychotherapists saw a slightly higher proportion of patients from neighbourhoods with the lowest income quintile. The regional pattern of patients was similar to that of physician practices, with patients seen by psychotherapists predominantly living in high-supply LHINs.

The 2 categories of psychiatrists had similar proportions of patients in the 4 groups with urgent need for mental health services. Primary care physician psychotherapists had a smaller proportion of patients in the 4 groups than the 2 categories of psychiatrists but a larger proportion than PCP nonpsychotherapists. However, in terms of absolute number of patients, PCP nonpsychotherapists saw the highest number of patients for mental health and addiction reasons, including those with urgent needs, followed by psychiatrist nonpsychotherapists. Primary care physician psychotherapists saw slightly more patients than psychiatrist psychotherapists, including more patients with 2 or more substance-related emergency department visits in the prior 12 months. However, psychiatrist psychotherapists saw more patients in the other 3 groups of patients with urgent need.

\section{Access to physicians for patients with urgent need for mental health services}

Table 4 shows the proportion of patients in the 4 groups of patients with urgent need who were seen within 30 and 180 days of the index event by physicians within each of the 4 categories. In each patient group, about half of the patients did not have any visit to a PCP or psychiatrist within 30 days, and one-quarter to one-third did not have any visit to a PCP or psychiatrist within 180 days. Primary care physician and psychiatrist nonpsychotherapists saw a similar proportion of 


\begin{tabular}{|c|c|c|c|c|c|c|}
\hline \multirow[b]{2}{*}{ Characteristic } & \multicolumn{2}{|c|}{ No. (\%) of physicians* } & \multirow[b]{2}{*}{ SDiff } & \multicolumn{2}{|c|}{ No. $(\%)$ of physicians ${ }^{*}$} & \multirow[b]{2}{*}{ SDiff† } \\
\hline & $\begin{array}{c}\text { PCP } \\
\text { psychotherapists } \\
n=404\end{array}$ & $\begin{array}{c}\text { PCP } \\
\text { nonpsychotherapists } \\
n=12368\end{array}$ & & $\begin{array}{c}\text { Psychiatrist } \\
\text { psychotherapist } \\
n=586\end{array}$ & $\begin{array}{c}\text { Psychiatrist } \\
\text { nonpsychotherapist } \\
n=1564\end{array}$ & \\
\hline Female sex & $206(51.0)$ & $5712(46.2)$ & 0.10 & $239(40.8)$ & $659(42.1)$ & 0.03 \\
\hline \multicolumn{7}{|l|}{ Age category, yr } \\
\hline$<30$ & $0(0.0)$ & $136(1.1)$ & 0.15 & $0(0.0)$ & $0(0.0)$ & - \\
\hline 30-39 & $24(5.9)$ & $2173(17.6)$ & 0.37 & $16(2.7)$ & 208 (13.3) & 0.40 \\
\hline $40-49$ & $47(11.6)$ & $2628(21.2)$ & 0.26 & $49(8.4)$ & $386(24.7)$ & 0.45 \\
\hline $50-59$ & $121(30.0)$ & $3038(24.6)$ & 0.12 & $146(24.9)$ & $372(23.8)$ & 0.03 \\
\hline$\geq 60$ & $204(50.5)$ & $2992(24.2)$ & 0.57 & $367(62.6)$ & $443(28.3)$ & 0.73 \\
\hline Missing & $8(2.0)$ & 1401 (11.3) & 0.38 & $8(1.4)$ & $155(9.9)$ & 0.38 \\
\hline Age, yr, mean \pm SD & $59.8 \pm 11.5$ & $51.2 \pm 12.3$ & 0.73 & $63.0 \pm 11.0$ & $52.9 \pm 11.8$ & 0.88 \\
\hline Full-time & $218(54.0)$ & $8721(70.5)$ & 0.35 & $417(71.2)$ & $1088(69.6)$ & 0.03 \\
\hline \multicolumn{7}{|l|}{ Medical school } \\
\hline University of Toronto & $115(28.5)$ & $2615(21.1)$ & 0.17 & $148(25.3)$ & $185(11.8)$ & 0.35 \\
\hline University of Ottawa & $34(8.4)$ & $914(7.4)$ & 0.04 & $23(3.9)$ & $82(5.2)$ & 0.06 \\
\hline Queen's University & $25(6.2)$ & $656(5.3)$ & 0.04 & $17(2.9)$ & $82(5.2)$ & 0.12 \\
\hline McMaster University & $65(16.1)$ & $1104(8.9)$ & 0.22 & $47(8.0)$ & $145(9.3)$ & 0.04 \\
\hline Western University & $38(9.4)$ & $1140(9.2)$ & 0.01 & $31(5.3)$ & $76(4.9)$ & 0.02 \\
\hline Northern Ontario & $0(0.0)$ & $74(0.6)$ & 0.11 & $0(0.0)$ & $0(0.0)$ & - \\
\hline Other Canadian & $60(14.8)$ & $1433(11.6)$ & 0.10 & $138(23.5)$ & $251(16.0)$ & 0.19 \\
\hline Non-Canadian & $67(16.6)$ & 4432 (35.8) & 0.45 & $182(31.1)$ & $743(47.5)$ & 0.34 \\
\hline \multicolumn{7}{|l|}{ LHIN region of practice $\ddagger$} \\
\hline High supply & $178(44.1)$ & 2951 (23.9) & 0.44 & $401(68.4)$ & $632(40.4)$ & 0.59 \\
\hline Medium supply & $77(19.1)$ & $2515(20.3)$ & 0.03 & $63(10.8)$ & $363(23.2)$ & 0.34 \\
\hline Low supply & $149(36.9)$ & $6901(55.8)$ & 0.39 & $122(20.8)$ & $569(36.4)$ & 0.35 \\
\hline Missing & $0(0.0)$ & $1(0.0)$ & - & $0(0.0)$ & $0(0.0)$ & - \\
\hline $\begin{array}{l}\text { Note: LHIN = Local Health Int } \\
{ }^{*} \text { Except where noted otherwi } \\
\text { †A standardized difference of } \\
\text { fBased on whether the per } \\
\text { below the provincial average }\end{array}$ & $\begin{array}{l}\text { n Network, PCP }=\text { prin } \\
\text { than } 0.1 \text { is considere } \\
\text { pply of psychiatrists is } \\
\text { pply). }{ }^{9}\end{array}$ & $\begin{array}{l}\text { y care physician, } S D=\text { stan } \\
\text { linically significant.12 } \\
\text { uch higher than the provinc }\end{array}$ & d deviatic & $\begin{array}{l}\text { Diff }=\text { standardized di } \\
\text { supply), at or near th }\end{array}$ & $\begin{array}{l}\text { ence. } \\
\text { ovincial average (medium }\end{array}$ & ly), or \\
\hline
\end{tabular}

patients, about one-quarter, with the exception of the patients with 2 or more emergency department visits for substancerelated issues: PCP nonpsychotherapists saw more than twice as many of these patients as did psychiatrist nonpsychotherapists (29.8\% v. 13.9\%). Primary care physician and psychiatrist psychotherapists saw very few patients with urgent need for mental health services $(2 \%-3 \%)$.

\section{Interpretation}

There were just under 1000 physicians with practices focused on psychotherapy in Ontario in 2015/16, about $60 \%$ of whom were psychiatrists. The psychotherapist physicians were older than their nonpsychotherapist colleagues and were clustered in 2 large cities (Toronto and Ottawa). They saw a much smaller number of patients and saw them much more frequently. They served only a very low proportion $(1 \%-3 \%)$ of patients with urgent need for mental health services.

Our findings address the question of whether physician psychotherapists can meet the population need for psychotherapy in Ontario. Using a conservative 1-year prevalence of major depressive disorder of $4 \%^{13}$ and the number of Ontarians more than 14 years of age in 2016 (slightly more than 11 million $^{14}$ ), there were about 450000 people with major depressive disorder. With at least a similar number with anxiety disorders or adjustment disorders, one can estimate that at least 900000 Ontarians could have benefitted from psychotherapy. Psychotherapist physicians saw about 176000 patients in 2015/16, including a substantial number of patients seen only once. The existing complement of Ontario psychotherapist physicians cannot meet the need for psychotherapy for common mental disorders. 


\begin{tabular}{|c|c|c|c|c|c|c|}
\hline Variable & $\begin{array}{c}\text { PCP } \\
\text { psychotherapists } \\
n=404\end{array}$ & $\begin{array}{c}\text { PCP } \\
\text { nonpsychotherapists } \\
n=12368\end{array}$ & SDiff & $\begin{array}{c}\text { Psychiatrist } \\
\text { psychotherapists } \\
n=586\end{array}$ & $\begin{array}{c}\text { Psychiatrist } \\
\text { nonpsychotherapists } \\
n=1564\end{array}$ & SDiff \\
\hline \multicolumn{7}{|l|}{ No. of outpatients } \\
\hline Mean (SDiff†) & $218.27(300.20)$ & 1425.64 (1416.39) & 1.18 & $131.76(165.34)$ & $274.40(286.26)$ & 0.61 \\
\hline \multicolumn{7}{|l|}{ No. of new outpatients } \\
\hline Mean \pm SD & $125.12 \pm 219.42$ & $869.31 \pm 1161.39$ & 0.89 & $55.74 \pm 85.64$ & $162.06 \pm 200.36$ & 0.69 \\
\hline Median (IQR) & $40(14-146)$ & $516(278-939)$ & 1.65 & $26(9-69)$ & $107(41-210)$ & 0.95 \\
\hline \multicolumn{7}{|l|}{$\begin{array}{l}\text { Annual no. of visits per } \\
\text { outpatient }\end{array}$} \\
\hline \multicolumn{7}{|l|}{$\begin{array}{l}\text { Annual no. of visits per } \\
\text { patient, no. (\%) of } \\
\text { physicians }\end{array}$} \\
\hline$\leq 3$ & $112(27.7)$ & 10792 (87.3) & 1.51 & $77(13.1)$ & $998(63.8)$ & 1.22 \\
\hline $4-16$ & $244(60.4)$ & $1475(11.9)$ & 1.17 & $340(58.0)$ & $534(34.1)$ & 0.49 \\
\hline$\geq 17$ & 48 (11.9) & $101(0.8)$ & 0.47 & $169(28.8)$ & $32(2.0)$ & 0.80 \\
\hline $\begin{array}{l}\text { No. of single-visit } \\
\text { outpatients, mean (SDiff }+ \text { ) }\end{array}$ & $84.23(183.55)$ & 772.25 (1051.97) & 0.91 & 31.95 (51.62) & $117.78(158.94)$ & 0.73 \\
\hline $\begin{array}{l}<200 \text { outpatients annually, } \\
\text { no. (\%) of physicians }\end{array}$ & $275(68.1)$ & $1383(11.2)$ & 1.43 & $478(81.6)$ & $810(51.8)$ & 0.67 \\
\hline $\begin{array}{l}<100 \text { outpatients annually, } \\
\text { no. }(\%) \text { of physicians }\end{array}$ & $198(49.0)$ & $917(7.4)$ & 1.04 & $350(59.7)$ & $441(28.2)$ & 0.67 \\
\hline
\end{tabular}

Based on the observed proportion of patients with urgent mental health need who accessed psychotherapist physicians, a very small proportion of patient populations with urgent need have access to psychotherapist physicians compared to nonpsychotherapist physicians. As has been discussed in prior studies on psychiatrist supply in Ontario, ${ }^{9,15}$ the current Ontario Health Insurance Plan payment process is entirely fee-for-service and does not stipulate limits on visit frequency or the type of patient who should receive services. This may explain the low-volume practices where patients are seen with high frequency. The low rate of access observed more generally (e.g., $50 \%$ and $60 \%$ of people with emergency department visits for self-harm or substance use, respectively, saw no physician within $30 \mathrm{~d}$ of their emergency department discharge) suggests a broader problem of access to mental health services.

Even if financial incentives were better aligned with population need, publicly funded physician psychotherapists will most likely not meet the need for evidence-based psychotherapy. Other jurisdictions have developed system responses to meet this need. In England, Improving Access to Psychological
Therapies is now a well-established system that uses teams of trained lay therapists and measurement-based care processes to provide evidence-based psychotherapies such as cognitive behavioural therapy and interpersonal psychotherapy ${ }^{16}$ for a variety of mental disorders. ${ }^{17}$ In the United States, health maintenance organizations run by private insurance companies employ nonphysicians to provide evidence-based psychotherapy, with psychiatrists largely providing consultations and pharmacotherapy. ${ }^{18}$ These are all examples that improve access in some capacity but have limitations. The need for innovation to improve access to and quality of mental health care is urgent.

The Ontario government is in the midst of a pilot project that is based on a model of a population-based psychotherapy intervention that has been evaluated extensively and shown to be successful. ${ }^{16}$ This model of service delivery adheres to evidence and uses nonphysician psychotherapists. It may increase access to publicly funded psychotherapy equitably and efficiently.

\section{Limitations}

This study focused on outpatient visits among PCPs and psychiatrists. It has both strengths and limitations. Strengths 


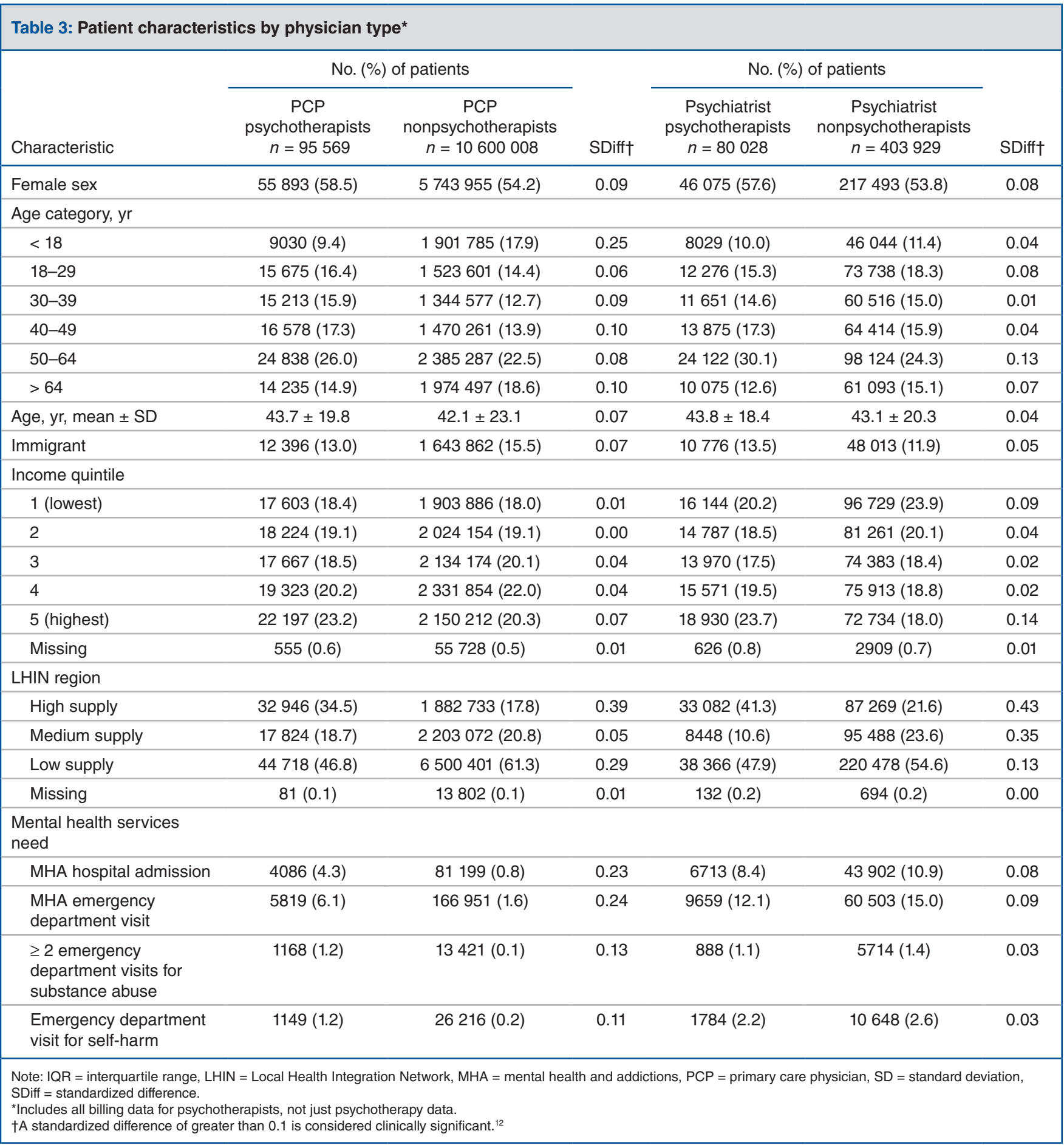

include the use of population-based data that captured all physicians in Ontario providing psychotherapy and the patients they served.

There are several limitations to this approach. Registered (nonphysician) psychotherapists are an alternative to physician psychotherapists but are not included in this study. Some of these nonphysician psychotherapists work in hospital and community settings, where they are paid with funds from the Ontario Ministry of Health and Long-
Term Care and thus provide therapy free of charge to patients. We were unable to determine the scope of this supply of psychotherapy in Ontario. Thus, our study underestimates the total supply of publicly funded psychotherapists in Ontario, but we do not believe the number of these therapists is large enough to invalidate our main findings.

Second, we do not know what type of psychotherapies were provided by physician psychotherapists. It is likely that 


\begin{tabular}{|c|c|c|c|c|}
\hline \multirow[b]{2}{*}{ Variable } & \multicolumn{4}{|c|}{ Index event, no. (\%) of patients } \\
\hline & $\begin{array}{l}\text { MHA hospital } \\
\text { admission } \\
n=96247\end{array}$ & $\begin{array}{c}\text { MHA emergency } \\
\text { department visit } \\
n=192641\end{array}$ & $\begin{array}{c}\text { Emergency } \\
\text { department visit for } \\
\text { self-harm } \\
n=28699\end{array}$ & $\begin{array}{c}\geq 2 \text { emergency } \\
\text { department visits } \\
\text { for substance use } \\
n=16022\end{array}$ \\
\hline \multicolumn{5}{|l|}{ Visit within $30 \mathrm{~d}$ of index event* } \\
\hline Visit to psychiatrist psychotherapist & $3004(3.1)$ & $4232(2.2)$ & $835(2.9)$ & $187(1.2)$ \\
\hline Visit to psychiatrist nonpsychotherapist & $27625(28.7)$ & $44736(23.2)$ & $8311(29.0)$ & $2224(13.9)$ \\
\hline Visit to PCP psychotherapist & $1969(2.0)$ & $2443(1.3)$ & $450(1.6)$ & $436(2.7)$ \\
\hline MHA visit to PCP nonpsychotherapist & $26465(27.5)$ & $53565(27.8)$ & $8098(28.2)$ & $4658(29.1)$ \\
\hline None of the above & $47329(49.2)$ & $104563(54.3)$ & $14254(49.7)$ & $9654(60.2)$ \\
\hline \multicolumn{5}{|l|}{ Visit within $180 \mathrm{~d}$ of index event* } \\
\hline Visit to psychiatrist psychotherapist & $5430(5.6)$ & $8759(4.6)$ & $1612(5.6)$ & $547(3.4)$ \\
\hline Visit to psychiatrist nonpsychotherapist & $45694(47.5)$ & $73462(38.1)$ & $12780(44.5)$ & $4707(29.4)$ \\
\hline Visit to PCP psychotherapist & $3835(4.0)$ & $5372(2.8)$ & $1016(3.5)$ & $1153(7.2)$ \\
\hline MHA visit to PCP nonpsychotherapist & $45817(47.6)$ & $92053(47.8)$ & $14492(50.5)$ & $8655(54.0)$ \\
\hline None of the above & $27228(28.3)$ & $65470(34.0)$ & 8367 (29.2) & $5553(34.7)$ \\
\hline
\end{tabular}

some of these psychotherapies were unstructured and did not adhere to an evidence-based framework, which would further reduce the impact of and access to psychotherapies.

Third, we do not know which patients from the 4 groups with urgent need would benefit from access to psychotherapy. However, we believe that a proportion far greater than the 1 in 33 to 1 in 85 who were seen by physician psychotherapists would benefit from evidence-based psychotherapies.

Finally, with respect to data quality, the immigration data we used have not been validated but, as an administrative data source, are likely to accurately capture relevant information on immigrant and refugee status for the majority of Ontario residents.

\section{Conclusion}

Evidence-based psychotherapy should be available to patients with the most prevalent mental disorders. Our findings suggest that there are far too few publicly funded physician psychotherapists in Ontario to meet the needs of these patients. Moreover, existing physician psychotherapists are not equitably distributed geographically in the province, and most have adopted practice styles that further impede access. The Coalition of Ontario Psychiatrists' recommendation to increase the number of psychiatrists in Ontario, although relevant to areas with critical shortages, will not address the existing poor access to psychotherapy. Our findings suggest a need to develop initiatives to increase access to psychotherapy that integrate the existing physician psychotherapists and adopt innovations that have been proven to improve access.

\section{References}

1. Lopez AD, Mathers C, Ezzati M, et al. Global and regional burden of disease and risk factors, 2001: systematic analysis of population health data. Lancet 2006;367:1747-57

2. Baxter AJ, Vos T, Scott KM, et al. The global burden of anxiety disorders in 2010. Psychol Med 2014;44:2363-74.

3. Parikh SV, Quilty LC, Ravitz P, et al. Canadian Network for Mood and Anxiety Treatments (CANMAT) 2016 clinical guidelines for the management of adults with major depressive disorder: section 2. Psychological treatments. Can f Psychiatry 2016;61:524-39.

4. Depression in adults: recognition and management. London (UK): National Institute for Health and Care Excellence; 2009.

5. Generalized anxiety disorder and panic disorder in adults: management. London (UK): National Institute for Health and Care Excellence; 2011.

6. Low C. Q \& A about training in medical psychotherapy through the Medical Psychotherapy Association Canada (MDPAC). Newmarket (ON): Medical Psychotherapy Association Canada; 2017. Available: https://www.mdpac.ca/ Training_to_Become_an_MD_Psychotherapist.html (accessed 2019 Dec. 6).

7. Coalition of Ontario Psychiatrists. Ontario needs psychiatrists: chronic psychiatry shortage contributing to Canada's mental bealth crisis. Toronto: Ontario Psychiatric Association; 2017.

8. Chiu M, Lebenbaum M, Lam K, et al. Describing the linkages of the Immigration, Refugees and Citizenship Canada permanent resident data and vital statistics death registry to Ontario's administrative health database. BMC Med Inform Decis Mak 2016;16:135.

9. Kurdyak P, Stukel T, Goldbloom D, et al. Universal coverage without universal access: a study of psychiatrist supply and practice patterns in Ontario. Open Med 2014;8:e87-99.

10. Full-time equivalent physicians report, fee-for-service physicians in Canada, 2004-2005. Ottawa: Canadian Institute for Health Information; 2006.

11. Steele LS, Glazier RH, Lin E, et al. Using administrative data to measure ambulatory mental health service provision in primary care. Med Care 2004;42: 960-5.

12. Austin P. Using the standardized difference to compare the prevalence of a binary variable between two groups in observational research. Commun Stat Simul Comput 2009;38:1228-34.

13. Patten SB, Williams JV, Lavorato DH, et al. Descriptive epidemiology of major depressive disorder in Canada in 2012. Can 7 Psychiatry 2015;60:23-30.

14. Census profile, 2016 census: Ontario (province) and Canada (country). Ottawa: Statistics Canada; 2019. Available: https://www12.statcan.gc.ca/census -recensement/2016/dp-pd/prof/details/Page.cfm?Lang=E\&Geo1=PR\&Code1= $35 \& \mathrm{Geo} 2=\&$ Code $2=\&$ Data $=$ Count $\&$ Search Text $=$ Ontario $\&$ Search Type $=$ Begin 
s\&SearchPR=01\&B1=All\&GeoLevel=PR\&GeoCode=35 (accessed 2019 Feb. 23).

15. Rudoler D, de Oliveira C, Cheng J, et al. Payment incentives for communitybased psychiatric care in Ontario, Canada. CMA7 2017;189:E1509-16.

16. Markowitz JC, Weissman MM. Interpersonal psychotherapy: principles and applications. World Psychiatry 2004;3:136-9.

17. Clark DM, Canvin L, Green J, et al. Transparency about the outcomes of mental health services (IAPT approach): an analysis of public data. Lancet 2018; 391:679-86.

18. Ma CA, McGuire TG. Costs and incentives in a behavioral health carve-out. Health Aff (Millwood) 1998;17:53-69.

Affiliations: Centre for Addiction and Mental Health (Kurdyak, Zaheer, Carvalho, de Oliveira, Stergiopoulos, Mulsant); Department of Psychiatry (Zaheer, Carvalho, Fefergrad, Stergiopoulos, Mulsant) and Institute for Health Policy, Management and Evaluation (de Oliveira), Faculty of Medicine, University of Toronto; ICES (Kurdyak, Lebenbaum, Wilton), Toronto, Ont.

Contributors: All of the authors contributed to the study conception and design, data analysis and interpretation, and drafting the manuscript, approved the final version to be published and agreed to be accountable for all aspects of the work.
Funding: This work was supported in part by the Medical Psychiatry Alliance, a collaborative health partnership of the University of Toronto, the Centre for Addiction and Mental Health, The Hospital for Sick Children, Trillium Health Partners, the Ontario Ministry of Health and Long-Term Care and an anonymous donor.

Disclaimer: This study was supported by ICES, which is funded by an annual grant from the Ontario Ministry of Health and Long-Term Care (MOHLTC). The opinions, results and conclusions reported in this paper are those of the authors and are independent from the funding sources. No endorsement by ICES or the Ontario MOHLTC is intended or should be inferred. Parts of this material are based on data and/or information compiled and provided by the Canadian Institute for Health Information (CIHI) and Immigration, Refugees and Citizenship Canada (IRCC). However, the analyses, conclusions, opinions and statements expressed in the material are those of the authors and not necessarily those of CIHI or IRCC.

Supplemental information: For reviewer comments and the original submission of this manuscript, please see www.cmajopen.ca/content/8/1/ E105/suppl/DC1. 